\title{
ON THE TORUS THEOREM FOR CLOSED 3-MANIFOLDS
}

BY

\author{
C. D. FEUSTEL( ${ }^{1}$ )
}

ABSTRACT. In this paper we give the appropriate generalization of the torus theorem to closed, sufficiently large, irreducible, orientable 3-manifolds.

I. Introduction. In [2] we proved the torus theorem for a bounded, orientable, 3-manifold $M$ and conjectured that the theorem would also hold if $M$ were sufficiently large, closed, irreducible, and orientable. W. Jaco has pointed out to the author that one can construct a counterexample to our conjecture by sewing a solid torus $M_{1}$ to the knot space $M_{2}$ of a torus knot so that the fibrings of $M_{1}$ and $M_{2}$ agree. Of course one requires that the spanning surface of the torus knot is of genus greater than one.

It is the purpose of this paper to prove that if $M$ is a sufficiently large, closed, orientable irreducible 3-manifold that admits an essential map of a torus, either $M$ admits an essential embedding of a torus or a finite sheeted covering space of $M$ has a particular structure. It would be interesting if all closed, orientable irreducible 3-manifolds that admit essential maps of tori and not essential embeddings of tori have covering spaces with this structure. In particular such 3-manifolds would be "almost sufficiently large."

Theorem 1 also aims at a partial answer to question 3 in [4] for genus 1 surfaces.

The results of this paper also follow from theorems proved independently by Johanssen and by Jaco and Shalen, which classify the boundary-preserving maps of a torus or annulus into a sufficiently large 3-manifold, up to boundarypreserving homotopy.

II. Notation. We adopt the notation and conventions in [7] without change. We let $A$ represent an annulus, $c_{1}$ and $c_{2}$ the components of $\partial A$ and $\alpha$ a spanning arc of $A$ (i.e. $A-\alpha$ is connected and simply connected) throughout this paper. Let $M$ be a 3-manifold and $F$ an incompressible surface in $\partial M$. Then $f:(A, \partial A) \rightarrow(M, F)$ is an F-essential map if

(1) $f_{*}: \pi_{1}(A) \rightarrow \pi_{1}(M)$ is monic.

(2) $f(\alpha)$ is not homotopic rel its boundary to an arc on $F$.

Received by the editors November 22, 1974 and, in revised form, April 18, 1975. AMS (MOS) subject classifications (1970). Primary 55A35; Secondary 55A05.

Key words and phrases. 3-manifold, essential map, torus.

(1) The author is partially supported by NSF Grant GP 15357. 
If $F=\partial M$, we say that $f$ is essential. Let $T$ be a torus. A map $f: T \rightarrow M$ is essential if

(1) $f_{*}: \pi_{1}(T) \rightarrow \pi_{1}(M)$ is monic.

(2) There is a loop $\lambda \subset T$ such that $f(\lambda)$ is not freely homotopic to a loop in $\partial M$. Note that the second condition does not apply if $M$ is closed.

Let $F$ be a surface embedded in $M$ or $\partial M$. Let $g_{1}, g_{2}:(A, \partial A) \rightarrow(M, F)$, then $g_{1}$ and $g_{2}$ are parallel rel $F$ if there is an embedding $H: A \times I \rightarrow M$ such that

(1) $H(A \times\{0\})=g_{1}(A)$,

(2) $H(A \times\{1\})=g_{2}(A)$,

(3) $H(\partial A \times I) \subseteq F$.

Let $M_{1}$ be a 3-submanifold of $M$. Then $\operatorname{Fr}\left(M_{1}, M\right)$, or simply $\operatorname{Fr}\left(M_{1}\right)$ when no confusion can result, is the closure of $\left(\partial M_{1} \cap(M-\partial M)\right)$.

III. Supporting results. The following results are useful in the proof of the principal theorem of this paper. Proposition 4 is of some interest in itself.

LEMMA 1. Let $M$ be a closed, connected, irreducible 3-manifold and $F$ a closed, connected, 2-sided, incompressible surface of minimal genus embedded in $M$. Let $N$ be a regular neighborhood of $F$ in $M$. Let $f: T \rightarrow M$ be an essential map. Suppose

(1) $f^{-1}(F)$ is the union of a nonempty collection of essential simple loops.

(2) $f$ is not homotopic to a map $\bar{f}: T \rightarrow M$ such that $\bar{f}^{-1}$ contains fewer components than $f^{-1}(F)$.

(3) The $f$ image of each component of $T-f^{-1}(F)$ meets both components of $N-F$.

Then there is an essential map $g: K \rightarrow M$ where $K$ is either a torus or a Klein bottle such that

(1) $g^{-1}(F)$ is the union of a nonempty collection of essential simple loops.

(2) $K-g^{-1}(F)$ is the union of a collection of open annuli.

(3) The restriction of $g$ to the closure of each component of $K-g^{-1}(F)$ is not homotopic $\mathrm{rel} \mathrm{g}^{-1}(F)$ to a map into $F$.

(4) The restriction of $g$ to each component of $g^{-1}(F)$ and $K-g^{-1}(F)$ is a homeomorphism.

ProOF. This is Lemma 5.11 in [2] except that $M$ is a closed manifold.

LEMMA 2. Let $F$ be a closed, connected, 2-sided, incompressible surface of minimal genus properly embedded in $M$. Let $N$ be a regular neighborhood of $F$ in $M$. Let $K_{1}$ be a torus or a Klein bottle and $f: K_{1} \rightarrow M$ an essential map such that

(1) $f^{-1}(F)$ is the union of a nonempty collection of disjoint simple loops. 
(2) The components of $K_{1}-f^{-1}(F)$ are open annuli whose closures we denote by $A_{1}, \ldots, A_{n}$.

(3) $f\left(A_{1}\right)$ meets only one component of $R-F_{1}$.

(4) $f \mid A_{i}$ is not homotopic rel $\partial A_{i}$ to a map into $F$ for $i=1, \ldots, n$.

(5) $f$ is not homotopic to a map $f_{1}$ such that $f_{1}^{-1}(F)$ contains fewer loops than $f^{-1}(F)$.

Then there is an essential embedding $g: T \rightarrow M$.

Proof. This is an immediate consequence of Lemma 5.12 in [2] since the boundary of a regular neighborhood of an essential embedding of a Klein bottle in $M$ is an essential torus.

Lemma 3. Let $M$ be a closed, irreducible, orientable 3-manifold and $F$ a closed, connected, 2-sided, nonseparating, incompressible surface embedded in $M$. If $M$ admits an essential map $f: T \rightarrow M$, either

(1) $M$ admits an essential embedding $g: T \rightarrow M$ or

(2) $M$ is a fibre space with base $S^{1}$ and fibre $F$ and there is a finite sheeted cyclic cover $(\widetilde{M}, p)$ of $M$ associated with $F$ such that $\widetilde{M}$ is homeomorphic to $F \times S^{1}$.

Proof. After the usual argument, we suppose that $f^{-1}(F)$ is the union of a collection of disjoint, essential, simple loops. If $f^{-1}(F)$ is empty, it is a consequence of Theorem 8 in [2] that there is an embedding $g: T \rightarrow M-F$ such that $g_{*}: \pi_{1}(T) \rightarrow \pi_{1}(M-F)$ is an injection. Since $\pi_{1}(M-F) \rightarrow \pi_{1}(M)$ is monic, $g: T \rightarrow M$ is an essential map. Thus we may suppose that $f^{-1}(F)$ is the union of a nonempty collection of disjoint simple essential loops $\lambda_{1}, \ldots, \lambda_{n}$ and that the number of loops in this collection cannot be reduced by a homotopy of $f$.

Let $N$ be a regular neighborhood of $F$ in $M$. If the $f$ image of some component of $T-f^{-1}(F)$ meets both components of $N-F$, Lemma 3 is an immediate consequence of Lemma 2. Thus we may suppose that the $f$ image of each component of $T-f^{-1}(F)$ meets both components of $N-F$. It is now a consequence of Lemma 1 that there is an essential map $f: K \rightarrow M$ where $K$ is either a torus or a Klein bottle such that

(1) $f^{-1}(F)$ is the union of a nonempty collection of disjoint simple essential loops.

(2) Each component of $K-f_{1}^{-1}(F)$ is an open annulus.

(3) The restriction of $f_{1}$ to each component of $K-f_{1}^{-1}(F)$ and $f_{1}^{-1}(F)$ is a homeomorphism.

(4) $f_{1}$ is not homotopic to a map $\bar{f}_{1}$ such that $\bar{f}_{1}^{-1}(F)$ has fewer components than $f_{1}^{-1}(F)$. 
If the $f_{1}$ image of some component of $K-f_{1}^{-1}(F)$ meets only one component of $N-F$, we apply Lemma 2 to complete the proof of Lemma 3. If $f_{1}^{-1}(F)$ is a single loop, $f_{1}$ is an embedding and we are finished. Let $A_{1}, \ldots, A_{n}$ be the closures of the components of $K-f_{1}^{-1}(F)$ and $\lambda_{1}, \ldots, \lambda_{n}$ the components of $f_{1}^{-1}(F)$. We may now suppose that $f_{1}\left(A_{i}-\partial A_{i}\right)$ meets both components of $N-F$ for $i=1, \ldots, n$ where $n \geqslant 2$.

We suppose that $f$ has been chosen so that the number of loops in $f^{-1}(F)$ is minimal. Suppose $A_{i} \cap f^{-1}\left(A_{j}\right)$ contains an essential simple loop $\lambda$ where $1 \leqslant i<j \leqslant n$. Let $\lambda_{2} \subset A_{j}$ be a loop on $K$ such that $f\left(\lambda_{2}\right)=f\left(\lambda_{1}\right)$. Since $\lambda_{1}$ is essential, $\lambda_{2}$ is essential. Now $K-\left(\lambda_{1} \cup \lambda_{2}\right)$ is the union of two open annuli whose closures we denote by $B_{1}$ and $B_{2}$. We observe that $f\left(B_{1}\right)$ defines an essential map $\bar{f}$ of a closed connected surface $K^{1}$ of Euler characteristic zero such that $\bar{f}\left(K^{1}\right)=f\left(B_{1}\right), \bar{f}$ is essential by Lemma 5.3 in [2]. This is easily seen to contradict our assumption that $f$ was chosen so that $f^{-1}(F)$ would contain a minimal number of loops. Thus $A_{i} \cap f^{-1}\left(A_{j}\right)$ contains no essential simple loops for $1 \leqslant i, j \leqslant n$ and $i \neq j$.

For any essential map $\bar{f}: K \rightarrow M$ such that $\bar{f}^{-1}(F)=\bigcup_{i=1}^{n} \lambda_{i}$, we define $X(\bar{f})=\bigcup_{i \neq j}^{n} \bar{f}\left(\lambda_{i}\right) \cap \bar{f}\left(\lambda_{j}\right)$. We suppose that $f_{1}$ has been chosen to satisfy (1)(5) below and so that there is no map $\bar{f}$ homotopic to $f_{1}$ such that

(1) $\bar{f}^{-1}(F)=f_{1}^{-1}(F)=\bigcup_{i=1}^{n} \lambda_{i}$.

(2) $\bar{f} \mid \lambda_{i}$ is a homeomorphism for $i=1, \ldots, n$.

(3) $\bar{f} \mid\left(A_{i}-\partial A_{i}\right)$ is a homeomorphism for $i=1, \ldots, n$.

(4) The cardinality of $X(\bar{f})$ is less than that of $X\left(f_{1}\right)$.

(5) $\bar{f}\left(\lambda_{i}\right) \cap \bar{f}\left(\lambda_{j}\right) \cap \bar{f}\left(\lambda_{k}\right)=\varnothing$ if $1 \leqslant i<j<k \leqslant n$.

Now it can be seen that $X\left(f_{1}\right)$ is a finite set. We may assume that

$$
J_{i j}=\operatorname{cl}\left(A_{i} \cap f_{1}^{-1} f_{1}\left(\operatorname{int}\left(A_{j}\right)\right)\right)
$$

is the union of a collection of disjoint simple loops and arcs properly embedded in $A_{i}$ where $1 \leqslant i<j \leqslant n$.

We claim every arc in $J_{i j}$ properly embedded in $A_{i}$ is a spanning arc of $A_{i}$. Suppose $\beta_{1} \subseteq J_{i j}$ is an arc properly embedded in $A_{i}$ such that $\partial \beta_{1}$ lies on a single component of $\partial A_{i}$. Now $\beta_{1}$ cuts off a disk $D_{1} \subseteq A_{i}$. By construction $f_{1} \mid D_{1}$ is a homeomorphism. Let $\beta_{2}$ be the arc on $A_{j}$ such that $f_{1}\left(\beta_{2}\right)=f_{1}\left(\beta_{1}\right)$. Now $\beta_{2}$ cuts off a disk $D_{2} \subseteq A_{j}$ and $f_{1} \mid D_{2}$ is a homeomorphism. We may choose $D_{1}$ so that $f_{1}\left(D_{1}\right) \cap f_{1}\left(D_{2}\right)$ is the union of $f_{1}\left(\beta_{1}\right)$ and a collection (possibly empty) of disjoint simple loops properly embedded in $f_{1}\left(D_{1}\right)$. Let $\beta_{1}^{\prime}$ and $\beta_{2}^{\prime}$ be the closures of $\partial D_{1}-\beta_{1}$ and $\partial D_{2}-\beta_{2}$ respectively. Now $f_{1}\left(\beta_{1}^{\prime}\right) \cup f_{2}\left(\beta_{2}^{\prime}\right)$ is a simple loop $\lambda \subset \bigcup_{i=1}^{n} f_{1}\left(\lambda_{i}\right)$ and $\lambda$ is nullhomotopic in $M$ across the singular disk $f_{1}\left(D_{1}\right) \cup$ $f_{1}\left(D_{2}\right)$. Since $F$ is incompressible, we may suppose that $\lambda$ bounds a disk $D$ embedded in $F$. After the usual argument, we may suppose that each $\operatorname{arc} \beta \subset$ 
$\bigcup_{i=1}^{n} f_{1}\left(\lambda_{i}\right)$ properly embedded in $D$ meets $f_{1}\left(\beta_{j}^{\prime}\right)$ in a single point for $j=1,2$. Since $f_{1}\left(\beta_{1}^{\prime}\right)$ is isotopic rel its boundary to $f_{1}\left(\beta_{2}^{\prime}\right)$ across $D$ it can be seen that $f_{1}$ was not chosen so that $X\left(f_{1}\right)$ would be minimal.

Similarly if $\beta_{1}$ and $\beta_{2}$ are arcs in $\lambda_{i}$ and $\lambda_{j}$ where $1 \leqslant i<j \leqslant n$ and $f_{1}\left(\beta_{1}\right)$ $\cup f_{1}\left(\beta_{2}\right)$ bounds a disk $D \subseteq F$, we can stow that the best possible choice was not made for $f_{1}$.

Let $N_{1}^{\prime}$ be a regular neighborhood of $\left.\bigcup_{i=1}^{n} f_{1} \lambda_{i}\right)$ in $F$. Now $N_{1}^{\prime}$ is a 2 submanifold of $F$. Let $N_{1} \subseteq F$ be the smallest submanifold of $F$ that contains some given component of $N_{1}^{\prime}$ so that $\pi_{1}\left(N_{1}\right) \rightarrow \pi_{1}(F)$ is an injection. We claim that there is a submanifold $M_{1}$ of $M$ such that

(1) $M_{1}$ is a fibre bundle with base $S^{1}$ and fibre $N_{1}$.

(2) $N_{1}$ is a fibre of $M_{1}$.

(3) $\pi_{1}\left(M_{1}\right) \rightarrow \pi_{1}(M)$ is an injection.

In this case if $N_{1}$ has boundary, a component of $\partial M_{1}$ will be our desired essential embedding. Otherwise $M$ is a fibre bundle with base $S^{1}$ and fibre $F$.

Let $M^{*}$ be the manifold obtained by splitting $M$ along $F$ and $P: M^{*} \rightarrow M$ the natural projection map. We will assume that $N_{1}^{\prime}$ is connected; however, the interested reader will easily be able to fill in the missing details in the general case. Let $Q_{1}$ and $Q_{2}$ be the components of $P^{-1}\left(N_{1}\right)$. Clearly we need only show that there is an embedding of $H: N_{1} \times I \rightarrow M^{*}$ such that $H\left(N_{1} \times\{0\}\right)=$ $Q_{1}, H\left(N_{1} \times\{1\}\right)=Q_{2}$ and $P H\left(N_{1} \times I\right)=M_{1}$.

Let $h_{i}: A_{i} \rightarrow M^{*}$ be the map induced by $f_{1} \mid A_{i}$ for $i=1, \ldots, n$. Let $\mu_{i}=h_{i}\left(A_{i}\right) \cap Q_{1}$ and $\mu_{i}^{\prime}=h_{i}\left(A_{i}\right) \cap Q_{2}$ for $i=1, \ldots, n$. We may suppose that $\bigcup_{j=1}^{i} \mu_{j} \cap \mu_{i+1} \neq \varnothing$ for $i=1, \ldots, n-1$ since $N_{1}$ has been taken to be connected. Let $R_{1}$ be a regular neighborhood of $h_{1}\left(A_{1}\right)$. Now $h_{2}$ is homotopic to an embedding $h_{2}^{*}$ rel $\partial A_{2}$ so that the closure of $\left(\partial R_{1}-\left(Q_{1} \cup Q_{2}\right)\right) \cap h_{2}^{*}\left(A_{2}\right)$ is the union of a nonempty collection of disjoint simple arcs and loops. Since $\pi_{2}\left(M^{*}\right)$ $=0$ as a consequence of the irreducibility of $M$ and the sphere theorem [4], [8], $\partial R_{1} \cap h_{2}^{*}\left(A_{2}\right)$ may be taken to be a collection of disjoint simple arcs. We observe that each component $\gamma$ of $Q_{1} \cap R_{1} \cap h_{2}^{*}\left(\partial A_{2}\right)$ contains a crossing point of $h_{1}\left(\partial A_{1}\right)$ and $h_{2}\left(\partial A_{2}\right)$. Thus the component $\bar{D}$ of $h_{2}^{*}\left(A_{2}\right) \cap R_{1}$ containing $\gamma$ contains an arc running from $Q_{1}$ to $Q_{2}$. It is easily seen that $\bar{D}$ is either a disk or all of $h_{2}^{*}\left(A_{2}\right)$ and that $\bar{D} \cap\left(R_{1}-\left(Q_{1} \cup Q_{2}\right)\right)$ is a pair of disjoint simple arcs running from $Q_{1}$ to $Q_{2}$. Let $\bar{R}_{2}$ be a regular neighborhood of $R_{1} \cup h_{2}^{*}\left(A_{2}\right)$. It can be seen that $\bar{R}_{2}$ is homeomorphic to the product of $F_{2}=\bar{R}_{2} \cap Q_{1}$ with the unit interval. If $\pi_{1}\left(F_{2}\right) \rightarrow \pi_{1}\left(Q_{1}\right)$ is not an injection, there is a disk $E_{1}$ embedded in $Q_{1}$ such that $E_{1} \cap F_{2}=\partial E_{1}$. Now $\partial E_{1}$ is freely homotopic in $\partial \bar{R}_{2}$ to a loop in $Q_{2}$ so there is a disk $E_{2}$ embedded in $Q_{2}$ such that $E_{2} \cap \partial \bar{R}_{2}=\partial E_{2}$. Now $E_{1} \cup E_{2}$ together with an annulus in $\partial \vec{R}_{2}$ is a 2-sphere that bounds a 3-ball $B$ in $M^{*}$ and $B \cup \bar{R}_{2}$ is homeomorphic to the product of $F_{2} \cup E_{1}$ with the unit 
interval. We let $R_{2}$ be the smallest submanifold of $M^{*}$ such that

(1) $R_{2} \supseteq \bar{R}_{2}$.

(2) $R_{2}$ is homeomorphic to the product of $R_{2} \cap Q_{1}$ with the unit interval under a homeomorphism which carries $R_{2} \cap Q_{1}$ to $\left(R_{2} \cap Q_{1}\right) \times\{0\}$ and $\left(R_{2} \cap\right.$ $\left.Q_{2}\right)$ to $\left(R_{2} \cap Q_{1}\right) \times\{1\}$.

(3) $\pi_{1}\left(R_{2} \cap Q_{1}\right) \rightarrow \pi_{1}\left(Q_{1}\right)$ is an injection.

We admit that the proof above is more complicated than is necessary to find $R_{2}$, but the proof above also suffices to extend $R_{2}$ to $R_{3}$ when $h_{3}^{*}$ is chosen to be a map homotopic to $h_{3}$ rel $\partial A_{3}$ such that the closure of $\left(\partial R_{2}-\left(Q_{1} \cap Q_{2}\right)\right)$ $\cap h_{3}^{*}\left(A_{3}\right)$ is the union of a nonempty collection of disjoint simple arcs each of which runs from $Q_{1}$ to $Q_{2}$ and our claim follows after an inductive argument.

We assume that $M$ is a fibre space with base $S^{1}$ and fibre $F$ and observe that any finite sheeted covering associated with $F$ will also be such a fibre space. It follows from the proof above that $\pi_{1}(f(T) \cap F) \rightarrow \pi_{1}(F)$ is an epimorphism.

Let $(\widetilde{M}, p)$ be the $n$-sheeted cyclic cover of $M$ associated with $F$. Let $\widetilde{f}: K$ $\rightarrow \widetilde{M}$ be a lift of $f$. Note that $\widetilde{f}$ is an embedding. Let $\rho: \widetilde{M} \rightarrow \widetilde{M}$ be a generator of the group of covering translations of $\widetilde{M}$. Note that $\rho^{i}(K) \cap \rho^{j}(K)$ is the union of a collection of disjoint simple loops for $0 \leqslant i<j<n$. Let $m$ be twice the least common multiple of the intersection numbers of loops in $\rho^{i}(K) \cap \rho^{j}(K)$ and $F$ for $1 \leqslant i<j \leqslant n-1$. Let $\left(M^{\#}, q\right)$ be the $m$-sheeted cyclic cover of $\widetilde{M}$ associated with any component of $\rho^{-1}(F)$.

We claim that $M^{\#}$ is homeomorphic to $F \times S^{1}$. Observe that $q^{-1}\left(\rho^{i} \widetilde{f}(K)\right)$ is a torus embedded in $M^{*}$ for $0 \leqslant i<n$ and that each essential loop in $q^{-1}\left(\rho^{i} \widetilde{f}(K)\right) \cap q^{-1}\left(\rho^{j} \widetilde{f}(K)\right)$ meets each component of $(p q)^{-1}(F)$ in either the empty set or a singleton set.

Let $F^{\#}$ be a component of $(p q)^{-1}(F)$. We split $M^{\#}$ along $F^{\#}$ to obtain a 3-manifold $\bar{M}$ and let $P: \bar{M} \rightarrow M^{\#}$ be the natural projection map. Now $q^{-1}\left(\rho^{i} \widetilde{f}(K)\right)$ induces an embedding

$$
h_{i}:(A, \partial A) \rightarrow\left(\bar{M}, P^{-1}\left(F^{\#}\right)\right) \text { for } 0 \leqslant i<n .
$$

By construction $h_{i}(A) \cap h_{j}(A)$ is the union of a collection of disjoint simple spanning arcs and inessential simple loops for $0 \leqslant i<j<n$. Let $\bar{F}_{1}$ and $\bar{F}_{2}$ be the components of $P^{-1}\left(F^{\#}\right)$. We may suppose that $h_{i}\left(c_{1}\right) \subseteq \bar{F}_{1}$ for $0 \leqslant i<n$ and that $\bigcup_{i=1}^{k} h_{i}\left(c_{1}\right) \cap h_{k+1}\left(c_{1}\right)$ is not empty for $1 \leqslant k<n-1$ since we have shown that $f(K) \cap F$ is connected or $M$ admits an essential embedding of $T$.

Let $\bar{\alpha} \subset h_{1}(A) \cap h_{2}(A)$ be a spanning arc of $h_{1}(A)$. Observe that $P(\partial \alpha)$ is a single point. Let $h_{2}^{*}$ be a map homotopic to $h_{2}$ rel $\partial A$ such that $h_{2}^{*}(A) \cap h_{1}(A)$ contains no simple loops. Let $N_{1}$ be a regular neighborhood of $h_{2}^{*}\left(c_{1}\right) \cup h_{1}\left(c_{1}\right)$ in $\bar{F}_{1}$. Then there is an embedding $H_{1}: N_{1} \times I \rightarrow \bar{M}$ such that (1) $H_{1}(x, 0)=x$ for $x \in N_{1}$ and $H_{1}\left(N_{1} \times\{1\}\right) \subset \bar{F}_{2}$. 
(2) $P H_{1}(x, 0)=P H_{1}(x, 1)$ for $x \in N_{1}$.

(3) For each arc $\bar{\alpha} \subset h_{1}(A) \cap h_{2}^{*}(A), H_{1}^{-1}(\alpha)=\left\{x_{0}\right\} \times I$ for some $x_{0} \in$ $N_{1}$.

(4) $H_{1}^{-1} h_{1}(A)=h_{1}\left(c_{1}\right) \times I$.

(5) $H_{1}^{-1} h_{2}^{*}(A)=h_{2}\left(c_{1}\right) \times I$.

By assumption there is a spanning arc $\bar{\alpha}_{1}$ of $h_{3}(A)$ in $h_{1}(A) \cap h_{3}(A)$ or in $h_{2}^{*}(A) \cap h_{3}(A)$. We assume the former.

We claim that $H_{1}^{-1}\left(\bar{\alpha}_{1}\right)$ is homotopic rel its boundary in $H_{1}^{-1} h_{1}(A)$ to a product arc in $N_{1} \times I$. It follows from our claim that $h_{3}$ is homotopic rel $\partial A$ to a map $h_{3}^{*}$ such that $h_{3}^{*}(A) \cap h_{1}(A)$ is the union of a collection of disjoint simple spanning arcs and thus that $h_{3}^{*}(A) \cap h_{2}^{*}(A) \cap h_{1}(A)$ is empty. In this case we will be able to extend our product structure as was done in proving that $M$ is homeomorphic to a fibre space except that our product structure will be compatible with $P$ so that $\bar{M}$ can be seen to be $F \times S^{1}$.

It remains to establish our claim. Note that $P\left(\partial \bar{\alpha}_{1}\right)$ is a point so that $H_{1}^{-1}\left(\partial \bar{\alpha}_{1}\right)=\left\{x_{0}\right\} \times\{0,1\}$. Let $\theta: N_{1} \times I \rightarrow N_{1}$ be defined by $\theta(x, t)=x$ for $x \in N_{1}$. Since $P H_{1}(x, 0)=P H_{1}(x, 1)$ for $x \in N_{1}, \theta\left(H_{1}^{-1} h_{3}(\partial A)\right)$ contains an arc $\beta$ such that $H_{1}(\beta \times\{0,1\}) \subseteq h_{3}(\partial A)$ and $H_{1}(\beta \times\{0\})$ and $H_{1}(\beta \times\{1\})$ are the components of $h_{3}(\partial A) \cap H_{1}\left(N_{1} \times I\right)$ containing $\partial \bar{\alpha}_{1}$. Now $h_{3}$ is homotopic to a map $h_{3}^{\#}$ rel $\partial A \cup h_{3}^{-1}\left(\bar{\alpha}_{1}\right)$ such that $h_{3}^{\#-1} H_{1}\left(\partial N_{1} \times I\right)$ is a collection of disjoint spanning arcs. Let $D$ be the closure of the component of $A$ $h_{3}^{\#-1} H_{1}\left(\partial N_{1} \times I\right)$ that contains $h_{3}^{\#-1}\left(\bar{\alpha}_{1}\right)$. Now $\theta H_{1}^{-1} h_{3}^{\#}: D \rightarrow N_{1}$ determines a map $\phi:(A, \partial A) \rightarrow\left(N_{1}, \partial N_{1}\right)$. If $\phi\left(c_{1}\right)$ is nullhomotopic in $N_{1}, \theta H_{1}^{-1}\left(\bar{\alpha}_{1}\right)$ is nullhomotopic in $N_{1}$ and thus $H_{1}^{-1}\left(\bar{\alpha}_{1}\right)$ is homotopic to a product $\operatorname{arc}$ in $N_{1} \times I$. If $\phi\left(c_{1}\right)$ is essential in $N_{1}$, either $N_{1}$ is an annulus and $h_{1}\left(c_{1}\right)$ and $h_{2}\left(c_{2}\right)$ are isotopic in $N_{1}$ to disjoint loops or $\phi$ is homotopic to a map into $\partial N_{1}$ and $h_{3}\left(c_{1}\right)$ is isotopic in $\bar{F}_{1}$ to a loop not meeting $h_{1}\left(c_{1}\right) \cup h_{2}\left(c_{1}\right)$. Either of the above contradicts the minimality of the cardinality of $X(f)$ so our claim is established.

This completes the proof of Lemma 3.

REMARK 1. It is clear from the proof of Lemma 3 that if a manifold $M$ satisfying the conditions of Lemma 3 admits essential embeddings $g_{1}, g_{2}: T \rightarrow$ $M$ such that

(1) $g_{1}(T) \cap F$ and $g_{2}(T) \cap F$ are unions of collections of disjoint loops.

(2) The number of points in $F \cap g_{1}(T) \cap g_{2}(T)$ cannot be reduced by an isotopy of $g_{1}(T)$ or $g_{2}(T)$.

(3) $\pi_{1}\left(\left(g_{1}(T) \cup g_{2}(T)\right) \cap F\right) \rightarrow \pi_{1}(F)$ is onto (in particular $\left(g_{1}(T) \cup\right.$ $\left.g_{2}(T)\right) \cap F$ is connected), then $M$ has a finite sheeted covering homeomorphic to $F \times S^{1}$.

Proposition 4. Let $M$ be a compact, connected, irreducible 3-manifold 
and $F$ an incompressible surface in $\partial M$. Let $n \geqslant 1$ and for $i=1, \ldots, n f_{i}$ : $(A, \partial A) \rightarrow(M, F)$ be essential maps. Then there is an embedded line bundle $N$ in $M$ and collection of essential maps $\bar{f}_{1}, \ldots, \bar{f}_{n}:(A, \partial A) \rightarrow(M, F)$ such that

(1) $\bar{f}_{i}$ and $f_{i}:(A, \partial A) \rightarrow(M, F)$ are homotopic.

(2) $N \cap F$ is an incompressible surface in $M$ and is a 2-sheeted (not necessarily connected) cover of the zero section of $N$.

(3) $\partial N \cap \partial F$ contains $\bigcup_{i=1}^{n} \bar{f}_{i}(\partial A)$.

(4) $\operatorname{Fr}(N)$ is the union of a collection of essential annuli in $M$.

(5) No fiber of $N$ is homotopic rel its boundary to an arc in $F$.

PROOF. Let $h_{i}:(A, \partial A) \rightarrow(M, F)$ for $i=1, \ldots, m$ be a maximal collection of essential embeddings such that $h_{i}(A) \cap h_{j}(A)$ is empty for $1 \leqslant i<j \leqslant$ $m$ and $h_{i}(A)$ and $h_{j}(A)$ are not parallel rel $F$ for $1 \leqslant i<j \leqslant m$. It is a consequence of Theorem 3 in [1] that this collection is not empty and of the theorem on p. 60 in [7] that the collection is finite. Let $N_{1}$ be a regular neighborhood of $\bigcup_{i=1}^{m} h_{i}(A)$ in $M$. Then $N_{1}$ is homeomorphic to a line bundle. We suppose that the $f_{i}$ for $i=1, \ldots, n$ are in general position with respect to $\operatorname{Fr}\left(N_{1}\right)$ and that the number of points in $f_{i}^{-1}\left(\partial \operatorname{Fr}\left(N_{1}\right)\right)$ cannot be reduced by a homotopy of $f_{i}:(A, \partial A) \rightarrow(M, F)$ for $1 \leqslant i \leqslant n$.

Suppose that for some $j$, where $1 \leqslant j \leqslant n, f_{j}$ is not homotopic to a map $\bar{f}_{j}$ such that $f_{j}^{-1}\left(\partial \operatorname{Fr}\left(N_{1}\right)\right)$ is empty. Let $J=f_{j}^{-1}\left(\operatorname{Fr}\left(N_{1}\right)\right)$. Now $J$ is the union of a collection of disjoint simple arcs and loops properly embedded in $A$. Since $f_{i}^{*}: \pi_{1}(A) \rightarrow \pi_{1}(M)$ is monic and $\operatorname{Fr}\left(N_{1}\right)$ is incompressible in $M$, the usual argument shows that $J$ may be assumed to contain no nullhomotopic simple loops.

If $J$ contains an essential loop, each $\operatorname{arc} \beta_{1}$ in $J$ that is properly embedded in $A$ has its endpoints on a single component of $\partial A$. Let $D$ be the disk on $A$ cut off by $\beta_{1}$. Let $\beta_{2}$ be the closure of $\partial D-\beta_{1}$. We observe that $f_{i}\left(\beta_{1}\right)$ lies on a single component of $\operatorname{Fr}\left(N_{1}\right)$ and since the components of $\operatorname{Fr}\left(N_{1}\right)$ are essential annuli in $M, f_{j}\left(\partial \beta_{1}\right)$ lies on a single component of $\partial \operatorname{Fr}\left(N_{1}\right)$. But now $f_{j}\left(\beta_{1}\right)$ is homotopic rel its boundary to an arc in $\partial \operatorname{Fr}\left(N_{1}\right)$ as is $f_{j}\left(\beta_{2}\right)$ in $F$ since $F$ is incompressible. It can now be seen that $f_{j}$ was not chosen so that $f_{j}^{-1}\left(\partial \operatorname{Fr}\left(N_{1}\right)\right)$ would contain a minimal number of points. Thus $J$ can contain no essential loops.

The argument in the preceding paragraph also shows that each $\operatorname{arc} \beta \subseteq J$ and properly embedded in $A$ must be a spanning arc of $A$. It is not difficult to see that we may suppose that $f_{j}(\beta)$ is a spanning arc of one of the annuli in $\operatorname{Fr}\left(N_{1}\right)$ and further that $f_{j} \mid J$ is an embedding. Note that since $f_{j}(\partial A) \cap \partial \operatorname{Fr}\left(N_{1}\right)$ is not empty, $J$ contains a spanning arc of $A$.

Let $D$ be the closure of a component of $A-f_{j}^{-1}\left(N_{1}\right)$ and $N_{1}^{*}$ the closure of $M-N_{1}$. Observe that $D$ is a disk and $\partial D \cap f_{j}^{-1}\left(\operatorname{Fr}\left(N_{1}\right)\right)$ is the union of two spanning $\operatorname{arcs} \alpha_{1}$ and $\alpha_{2}$ of $A$. Let $\beta_{1}$ and $\beta_{2}$ be the closures of the components 
of $\partial D-\left(\alpha_{1} \cup \alpha_{2}\right)$. If $f_{j}(\partial D)$ is nullhomotopic in $\partial N_{1}^{*}, f_{j}\left(\beta_{1}\right)$ and $f_{j}\left(\beta_{2}\right)$ are homotopic in $F$ rel their boundaries to arcs in $\partial \operatorname{Fr}\left(N_{1}\right)$ and the best choice was not made for $f_{i}$. It is a consequence of the loop theorem [6] and its proof that there is a disk $D_{1}$ properly embedded in $N_{1}^{*}$ such that $\partial D_{1}$ is essential in $\partial N_{1}^{*}$ and $D_{1} \cap \operatorname{Fr}\left(N_{1}\right) \subseteq f_{j}\left(\beta_{1} \cup \beta_{2}\right)$. Since $F$ is incompressible and $\partial D_{1}-\operatorname{Fr}\left(N_{1}\right) \subseteq F$, $\partial D_{1} \cap \operatorname{Fr}\left(N_{1}\right)=f_{j}\left(\beta_{1} \cup \beta_{2}\right)$ and the arcs which are the closures of $\partial D_{1}-\operatorname{Fr}\left(N_{1}\right)$ are not homotopic in $F$ rel their boundaries to $\operatorname{arcs}$ in $N_{1} \cap F$. Now it can be seen that if $\bar{N}_{2}$ is a regular neighborhood of $N_{1} \cup D_{1}, \bar{N}_{2}$ is a (possibly twisted) line bundle.

It is a consequence of an inductive argument and the compactness of $F$ that there is a line bundle $N_{2} \subseteq M$ such that

(1) $N_{2} \cap F=N_{2} \cap \partial M$ is an incompressible surface in $M$ and is a 2-sheeted cover of the zero section of $N_{2}$.

(2) $\operatorname{Fr}\left(N_{2}\right)$ is the union of a nonempty collection of essential annuli in $M$.

(3) If $\bar{f}_{i}(\partial A) \cap N_{2}$ is not empty $\bar{f}_{i}(\partial A) \subseteq N_{2}$ for where $\bar{f}_{i}:(A, \partial A) \rightarrow$ $(M, F)$ is homotopic to $f_{i}$ for $i=1, \ldots, n$.

(4) No fibre of $N_{2}$ is homotopic rel its boundary to an arc in $F$.

We assume that the $\bar{f}_{i}$ were originally chosen as the $f_{i}$ so that we may omit the superscript on $f_{i}$ when we continue the argument.

Suppose that $f_{j}(\partial A)$ does not lie in $N_{2} \cap F$ and that $f_{j}\left(c_{1}\right)$ is not homotopic in $F$ to a loop in $N_{2} \cap F$. Let $F_{1}$ be the closure of the component of $F-N_{2}$ on which $f_{j}\left(c_{1}\right)$ lies. If $F_{1}$ is not the planar surface with three boundary components, it is a consequence of Theorem 2 in [3] and Theorem 1 in [1] that there is an essential embedding $h_{m+1}:(A, \partial A) \rightarrow(M, F)$ such that $h_{m+1}\left(c_{1}\right)$ lies on $F_{1}$ and $h_{m+1}\left(c_{1}\right)$ is not homotopic in $F_{1}$ to a component of $\partial F_{1}$. But it would follow that the collection $h_{1}, \ldots, h_{m}$ was not maximal. Thus if $F_{1}$ is the closure of any component of $F-N_{2}$ that contains a component of $f_{j}(\partial A)$, we may suppose that $F_{1}$ is the planar surface with three boundary components or an annulus and $f\left(c_{1}\right)$ or $f\left(c_{2}\right)$ is not homotopic in the closure of $F-N_{2}$ to a loop in $\partial\left(F \cap N_{2}\right)$.

Let $M_{1}$ be the closure of a component of $M-N_{2}$ such that $\left(M_{1} \cap F\right) \supset$ $F_{1} \supset f_{j}\left(c_{1}\right)$ where $F_{1}$ is a planar surface with three boundary components and $f_{j}\left(c_{1}\right)$ is not homotopic in $F_{1}$ to a loop in $N_{2} \cap F$. It is a consequence of Theorem 2 in [3], Theorem 1 in [2], and the maximality of the collection $h_{1}, \ldots$, $h_{m}$ that $N_{2} \supseteq \partial F_{1}$.

We claim (a) $M_{1}$ is a product line bundle. We show first that $\partial M_{1}$ must have total genus two and that $f_{j}(\partial A)$ does not lie entirely on $F_{1}$. Since the Euler characteristic of $F_{1} \subseteq \partial M_{1}$ is -1 , it is clear that the component $S$ of $\partial M_{1}$ containing $F_{1}$ has Euler characteristic at most -2 . Suppose $f_{j}(\partial A) \subseteq F_{1}$. Let $\lambda$ be a component of $\partial F_{1}$ such that $\lambda$ does not lie on an annular component of the 
closure of $S-F_{1}$. By Theorem 2 in [3] and Theorem 1' in [1] there is an essential (in $M$ ) embedding $h_{m+1}:(A, \partial A) \rightarrow\left(M, F_{1}\right)$ such that $h_{m+1}\left(c_{1}\right)=\lambda$. Now $h_{m+1}(A)$ is not parallel rel $F$ to $h_{i}(A)$ for $i=1, \ldots, m$ since then there would be an embedding $H: A \times I \rightarrow M$ such that $H(\partial A \times I) \subseteq F, H(A \times\{0\})$ $\subseteq M_{1}, H\left(c_{1} \times\{0\}\right)=\lambda, H(A \times\{1\}) \subseteq M-M_{1}$, and $H H^{-1}\left(\partial M_{1}\right)$ is an annulus from $\lambda$ to a second component of $\partial F_{1}$ or contains an incompressible nonannular 2-submanifold of $F_{1}$. Either of the above is impossible. Thus $f_{j}(\partial A)$ does not lie entirely on $F_{1}$.

Let $F_{2}$ be the component of $F \cap \partial M_{1}$ on which $f_{j}\left(c_{2}\right)$ lies. As above either $F_{2}$ is homeomorphic to $F_{1}$ or $f_{1}\left(c_{2}\right)$ is freely homotopic in $F_{2}$ to a loop in $\partial F_{2}$. It is a consequence of Theorem 2 in [3] and Theorem 1' in [1] that there are disjoint essential (in $M$ ) embeddings $g_{1}, g_{2}, g_{3}:(A, \partial A) \rightarrow\left(M_{1}, \partial M_{1}\right)$ such that $g_{1}\left(c_{1}\right) \cup g_{2}\left(c_{1}\right) \cup g_{3}\left(c_{1}\right)=\partial F_{1}$. Since $g_{1}$ is essential and $g_{1}(A) \cap \bigcup_{i=1}^{n} h_{i}(A)$ is empty we may suppose that $g_{1}(A)$ and $h_{1}(A)$ are parallel rel $F$. Similarly $g_{2}(A)$ and $g_{3}(A)$ are parallel rel $F$ to annuli in $\left\{h_{i}(A), i=1, \ldots, n\right\}$. It follows that $g_{1}\left(c_{2}\right) \cup g_{2}\left(c_{2}\right) \cup g_{3}\left(c_{2}\right)=\partial F_{2}$. Thus $S=F_{1} \cup F_{2} \cup g_{1}(A) \cup g_{2}(A) \cup g_{3}(A)$ and $F_{2}$ is homeomorphic to $F_{1}$.

It is a consequence of Theorem 2 in [3] and the existence of $f_{j}:(A, \partial A) \rightarrow$ $\left(M_{1}, \partial M_{1}\right)$ that there is a map $\bar{g}_{1}:(A, \partial A) \rightarrow\left(M_{1}, F_{1} \cup F_{2}\right)$ such that $\bar{g}_{1}\left(c_{1}\right)=$ $g_{1}\left(c_{1}\right), \bar{g}_{1}\left(c_{2}\right)$ is a component of $\partial F_{2}$, and $\bar{g}_{1}(\alpha)$ is homotopic rel its boundary to the product of an arc in $F_{1}, f_{j}(\alpha)$, and another arc in $F_{2}$. If $\bar{g}_{1}\left(c_{2}\right) \neq g_{1}\left(c_{2}\right)$, the collection $h_{1}, \ldots, h_{m}$ can be extended using Theorem 1 in [1] which is impossible. Now if $\bar{g}_{1}(\alpha)$ is not homotopic rel its boundary to an arc in $g_{1}(A)$, there is an $\left(g_{1}(A)\right)$-essential embedding $g_{1}^{\#}:(A, \partial A) \rightarrow\left(M_{1}, \partial M_{1}\right)$ such that $g_{1}^{\#}\left(c_{1}\right)=g_{1}\left(c_{1}\right)$ and $g_{1}^{\#}\left(c_{2}\right)=g_{1}\left(c_{2}\right)$ as a consequence of Theorem $1^{\prime}$. We observe that if $g_{1}^{\#}$ is not essential in $M, g_{1}$ is not essential in $M$, a contradiction; and if we deform $g_{1}^{\#}(\partial A)$ slightly into $F_{1} \cup F_{2}, g_{1}^{\#}(A)$ and $g_{1}(A)$ are not parallel rel $F$. Since $g_{1}^{\#}:(A, \partial A) \rightarrow(M, F)$ would extend the collection $h_{1}, \ldots, h_{m}$, this is impossible so $f_{j}(\alpha)$ is homotopic rel its boundary to the product of an arc in $F_{1}$ followed by a simple arc in $g_{1}(A)$, followed by an arc in $F_{2}$.

We assume that $f_{j}(\alpha) \subseteq \partial M_{1}$ as has been shown to be possible above and observe that $f_{j}:(A, \partial A) \rightarrow\left(M_{1}, \partial M_{1}\right)$ induces a map $f: D \rightarrow M$ such that $f(\partial D)$ $=f_{j}(\partial A \cup \alpha)$. If $f(\partial D)$ is nullhomotopic in $\partial M_{1}$, there is a map $f_{j}^{\prime}:(A, \partial A) \rightarrow$ $\left(M_{1}, \partial M_{1}\right)$ such that $f_{j}^{\prime}(A) \subseteq \partial M_{1}$ and $f_{j}\left|\partial A=f_{j}^{\prime}\right| \partial A$. It would follow that $f_{j}\left(c_{1}\right)$ is homotopic to a loop in $\partial F_{1}$.

We can now construct a disk $D_{1}$ properly embedded in $M_{1}$ such that $D_{1} \cap F_{i}$ is a simple arc properly embedded in $F_{i}$ for $i=1,2$ and $\partial D_{1}$ is not nullhomotopic in $\partial M_{1}$. Let $N_{3}$ be a regular neighborhood of $D_{1} \cup g_{1}(A)$. Now $N_{3}$ is a product line bundle and $f_{j}\left(c_{i}\right)$ is homotopic in $F_{i}$ to a loop in $g_{1}\left(c_{i}\right) \cup\left(D_{1} \cap F_{i}\right)$ for $i=$ 1,2. Using an argument similar to the one above, we can find disks $D_{2}$ and $D_{3}$ 
properly embedded in $M_{1}$ such that $D_{2} \cap F_{i}\left(D_{3} \cap F_{i}\right)$ is an arc properly embedded in $F_{i}$ for $i=1,2, D_{2} \cap \operatorname{Fr}\left(M_{1}\right) \subseteq g_{2}(A)\left(D_{3} \cap \operatorname{Fr}\left(M_{1}\right) \subseteq g_{3}(A)\right)$, and $\partial D_{2}$ and $\partial D_{3}$ are essential loops in $\partial M_{1}$. We may suppose that $\left(D_{1} \cap D_{2}\right) \cup\left(D_{2} \cap D_{3}\right)$ $\cup\left(D_{1} \cap D_{3}\right)$ is the union of a collection of disjoint simple arcs; and since $M_{1}$ is irreducible, it can be seen that $M_{1}$ is homeomorphic to $F_{1} \times I$. This establishes claim (a). Proposition 4 now follows since $M_{1} \cup N_{2}$ is a line bundle.

LEMMA 5. Let $M$ be a closed, irreducible, orientable 3-manifold and $F$ a closed, connected, 2-sided, separating, incompressible surface embedded in $M$. If $M$ admits an essential map $f: T \rightarrow M$, then either

(1) $M$ admits an essential embedding $g: T \rightarrow M$.

(2) The closures $M_{1}$ and $M_{2}$ of the components of $M-F$ are twisted line bundles.

Proof. We may suppose after the usual argument that $f^{-1}(F)$ is the union of a collection of disjoint simple loops. Since $\pi_{2}(M)=0$ and $F$ is incompressible, we may assume that no simple loop in $f^{-1}(F)$ is inessential in $T$. We suppose that $f$ has been chosen so that $f$ is not homotopic to a map $\bar{f}: T \rightarrow M$ such that $\bar{f}^{-1}(F)$ contains fewer loops than $f^{-1}(F)$. If $f^{-1}(F)$ is empty, Lemma 5 is an immediate consequence of Theorem 8 in [2] since $\pi_{1}\left(M_{j}\right) \rightarrow \pi_{1}(M)$ is monic for $j=1,2$.

Let $\bar{R}_{1}$ be a regular neighborhood of $f(T) \cap F$ in $F$. Let $R_{1}$ be the smallest 2-submanifold of $F$ such that $R_{1} \supseteq \bar{R}_{1}$ and $\pi_{1}\left(R_{1}\right) \rightarrow \pi_{1}(F)$ is monic. We suppose that $f$ has been chosen so that the Euler characteristic of $R_{1}$ will be maximal and so that $R_{1}$ has a maximal number of components. Let $A_{1}, \ldots, A_{n}$ be the closures of the components of $T-f^{-1}\left(M_{2}\right)$ and $f_{1}, \ldots, f_{n}$ be the restriction of $f$ to $A_{1}, \ldots, A_{n}$ respectively. It is a consequence of Proposition 3.3 in [2] that $f_{i}:\left(A_{i}, \partial A_{i}\right) \rightarrow\left(M_{1}, F\right)$ is an essential map for $i=1, \ldots, n$. It follows from Proposition 4 that there is a line bundle $N_{1}$ embedded in $M_{1}$ and maps $f_{i}:\left(A_{i}\right.$, $\left.\partial A_{i}\right) \rightarrow\left(M_{1}, F\right)$ homotopic to $f_{i}$ for $i=1, \ldots, n$ such that $\bigcup_{i=1}^{n} \bar{f}_{i}\left(\partial A_{i}\right) \subseteq$ $N_{1} \cap F$ where $N_{1} \cap F$ is an incompressible surface embedded in $R_{1}$. If $N_{1} \cap F$ is not a deformation retract of $R_{1}$, either $R_{1}$ has fewer components than $N_{1} \cap$ $F$ or the Euler characteristic of $N_{1} \cap F$ is greater than that of $N_{1}$. Either of the above contradicts our choice of $f$.

A similar argument shows that there is a line bundle $N_{2}$ embedded in $M_{2}$ such that $N_{2} \cap F$ is a deformation retract of $R_{1}$. Thus we may suppose that $N_{1} \cap F=N_{2} \cap F$. Note that if $N_{1} \cap F=F, \partial N_{1}=F$ is connected and both $N_{1}$ and $N_{2}$ are twisted line bundles.

Otherwise we observe that Proposition 4 guarantees that fibres of $N_{1}$ and $N_{2}$ are not homotopic rel their boundaries to arcs in $F$. Note that $N_{1} \cup N_{2}$ is a 3-submanifold $\bar{M}$ of $M$. We claim that $\partial \bar{M}$, which is the union of a collection of 
tori is incompressible in $M$. If not, there is a disk $D$ embedded in $M$ such that $D \cap \bar{M}=\partial D$. We may suppose that $D \cap F$ is the union of a collection of disjoint simple arcs. We suppose that $D$ has been chosen so that $D \cap F$ contains a minimal number of arcs. Let $D_{1}$ be a component of $D \cap M_{1}$ or $D \cap M_{2}$ such that $\partial D \cap D_{1}$ is connected. If $D=D_{1}, \partial D$ is freely homotopic in $\partial \bar{M}$ to a loop on $F$ and $N_{1} \cap F$ is not incompressible. Otherwise we suppose that $\partial D_{1} \cap N_{1}$ is not empty. If the arc $\partial D_{1} \cap N_{1}$ is not a spanning arc of the annular component of $\operatorname{Fr}\left(N_{1}\right)$ on which it lies, the number of arcs in $D \cap F$ could be reduced. Otherwise a fibre of $N_{1}$ is homotopic rel its boundary across $D_{1}$ to an $\operatorname{arc}$ in $F$. It follows that $\partial \bar{M}$ is incompressible in $M$ and each component of $\partial \bar{M}$ is an essential torus in $M$. This completes the proof of Lemma 5.

REMARK 2. It is clear from the proof of Lemma 5 that if $\pi_{1}(f(T) \cap F) \rightarrow$ $\pi_{1}(F)$ is not onto, $M$ admits an essential embedding $g: T \rightarrow M$.

IV. The principal theorem.

THEOREM 1. Let $M$ be a closed, connected, irreducible orientable 3-manifold. Let $f: T \rightarrow M$ be an essential map. Let $F$ be an incompressible surface embedded in $M$. Then either $M$ admits an essential embedding $g: T \rightarrow M$ or $M$ has a finite sheeted covering space homeomorphic to $F \times S^{\mathbf{1}}$. Furthermore if $M$ does not admit an essential embedding of $T, M$ is either a bundle with base $S^{1}$ and fibre $F$ or the union of two twisted line bundles each bounded by $F$.

Proof. We assume that $F$ has minimal genus. If $F$ does not separate $M$, Theorem 1 is an immediate consequence of Lemma 3. Otherwise we may assume that $M$ does not admit an essential embedding of a torus and by Lemma 5 the closures $M_{1}$ and $M_{2}$ of the components of $M-F$ will be twisted line bundles. Let $\sigma_{j}$ be an element of $\pi_{1}\left(M_{j}\right)$ so that $\sigma_{j} \notin \operatorname{im}\left(\pi_{1}(F) \rightarrow \pi_{1}\left(M_{j}\right)\right)$ for $j=1,2$ and $(\widetilde{M}, p)$ the covering space of $M$ associated with the subgroup $G$ of $\pi_{1}(M)$ generated by $\pi_{1}(F)$ and $\sigma_{1} \sigma_{2}$. Since $\sigma_{1}$ and $G$ generate $\pi_{1}(M)$ and $\pi_{1}(M) / \pi_{1}(F)$ $\cong Z, \widetilde{M}$ is a two-sheeted cover of $M$. It can be seen that neither component of $p^{-1}(F)$ separates $\widetilde{M}$. If $\widetilde{M}$ fails to admit an essential embedding of a torus, it is a consequence of Lemma 3 that $\widetilde{M}$ has a finite sheeted cover homeomorphic to $F \times S^{1}$.

Otherwise let $g: T \rightarrow \widetilde{M}$ be an essential embedding. Let $\rho: \widetilde{M} \rightarrow \widetilde{M}$ be the nontrivial covering translation. We may suppose that $g(T) \cap p^{-1}(F)$ is the union of a collection of disjoint, simple, essential loops since $g(T)$ and $p^{-1}(F)$ are incompressible in $\widetilde{M}$. We suppose further that $g(T)$ and $\rho g(T)$ are in general position and furthermore that if $\widetilde{F}$ is a component of $p^{-1}(F)$, one cannot decrease the number of points in $\widetilde{F} \cap g(T) \cap \rho g(T)$ via an isotopy $g(T)$ (and thus $\rho g(T)$ ). Now if $\pi_{1}(\rho g(T) \cap F) \rightarrow \pi_{1}(F)$ is not onto (in particular if $p g(T) \cap F$ is not connected), it follows from Remark 2 that $M$ admits an essential embedding. Otherwise 
by Remark $1, \tilde{M}$ (and thus $M$ ) has a finite sheeted cover homeomorphic to $F \times$ $S^{1}$. This completes the proof of Theorem 1 .

\section{REFERENCES}

1. James W. Cannon and C. D. Feustel, Essential embeddings of annuli and Mobius bands in 3-manifolds, Trans. Amer. Math. Soc. 215 (1976), 219-239.

2. C. D. Feustel, On the torus theorem and its applications, Trans. Amer. Math. Soc. 217 (1976), 1-43.

3. - Two extensions of the essential annulus theorem (submitted).

4. C. D. Papakyriakopoulos, On Dehn's lemma and the asphericity of knots, Ann. of Math. (2) 66 (1957), 1-26. MR 19, 761.

5. —, On solid tori, Proc. London Math. Soc. (3) 7 (1959), $281-299$. \#12526.

6. J. Stallings, On the loop theorem, Ann. of Math. (2) 72 (1960), 12-19. . MR 22

7. F. Waldhausen, On irreducible 3-manifolds which are sufficiently large, Ann. of Math. (2) 87 (1968), 56-88. MR 36 \#7146.

8. J. H. C. Whitehead, On 2-spheres in 3-manifolds, Bull. Amer. Math. Soc. 64 (1958), 161-166. MR 21 \#2241.

DEPARTMENT OF MATHEMATICS, VIRGINIA POLYTECHNIC INSTITUTE AND STATE UNIVERSITY, BLACKSBURG, VIRGINIA 24061 